\title{
Insularity induces changes on body and mandible morphology in a Mediterranean population of the greater white-toothed shrew Crocidura russula (Hermann, 1780)
}

\author{
Alejandro Sánchez-Chardi ${ }^{1,2^{*}}$, Marian García-Pando ${ }^{1^{*}}$, Eudald Pujol-Buxó ${ }^{1,3}$, Maria A. Sans-Fuentes ${ }^{4,5}$, María José \\ López-Fuster ${ }^{1,3}$, Francesc Muñoz-Muñoz ${ }^{6,7}$ \\ ${ }^{1}$ Departament de Biologia Evolutiva, Ecologia i Ciències Ambientals, Facultat de Biologia, Universitat de \\ Barcelona, Av. Diagonal 643, E-08028 Barcelona, Spain \\ ${ }^{2}$ Servei de Microscòpia, Facultat de Biociències, Universitat Autònoma de Barcelona, E-08193 Bellaterra \\ (Cerdanyola del Vallès), Barcelona, Spain \\ ${ }^{3}$ Institut de Recerca de la Biodiversitat (IRBio), Facultat de Biologia, Universitat de Barcelona, Av. Diagonal 643, \\ E-08028 Barcelona, Spain \\ ${ }^{4}$ Rhostat Consulting LLC, Tucson, Arizona 85705, USA \\ ${ }^{5}$ Statistics-GIDP. University of Arizona, Tucson AZ 85721, USA. \\ ${ }^{6}$ Departament de Biologia Animal, de Biologia Vegetal i d'Ecologia, Universitat Autònoma de Barcelona, E-08193 \\ Bellaterra (Cerdanyola del Vallès), Barcelona, Spain \\ ${ }^{7}$ E-mail: francesc.munozm@uab.cat \\ * These authors contributed equally to the study
}

Keywords: body condition, body size, island syndrome, mandible form, mechanical potential, Medes Islands, Baix Ter Natural Park, Spain

\begin{abstract}
Island populations of terrestrial mammals often undergo extensive behavioural and morphological changes when separated from mainland populations. Within small mammals these changes have been mainly reported in rodents but were poorly assessed in soricomorphs. In this study we compared mandible morphology and body condition between mainland and island populations of the greater white-toothed shrew, Crocidura russula. The results indicated that island specimens were bigger and heavier than the mainland counterpart, and they showed changes in mandible shape that were associated with higher mechanical potentials. We suggest that these changes might be the result of the interaction of two main factors taking place in the island population: ecological release (i.e. the decrease of predation and interspecific competition), and consequently the increase of intraspecific competition. While the increase in size and body condition in island shrews could be a direct result from reduced predation and interspecific competition, the changes in mandible shape and the increase of both mechanical potential and sexual dimorphism could have arisen indirectly as a response to stronger intraspecific competition.
\end{abstract}

\section{Contents}

Introduction .275

Material and methods

Study sites and sample collection...
Body mass, body length and body condition ....................277

Acquisition of mandible form data ...................................278

Analyses of mandible size and shape ...............................278

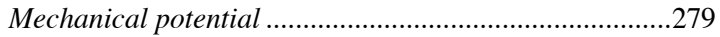

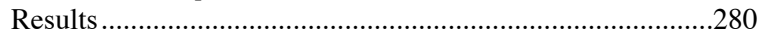

Body mass, body length and body condition index..........280

Mandible size and shape .................................................28

Mechanical potential .....................................................281

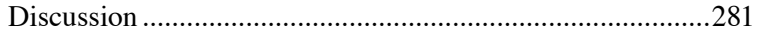

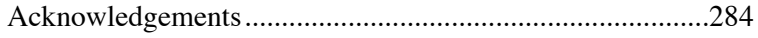

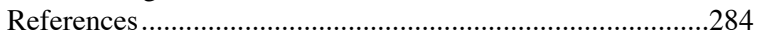

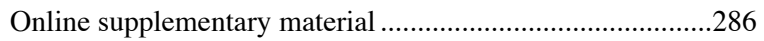

\section{Introduction}

Island ecosystems are characterized by simple biological communities having fewer species and wider ecological niches than their mainland counterparts. The decrease in species richness means a decrease in the diversity of predators and competitors, which allows for an increase in population density (Adler and Levins, 1994). These changes produce a cascade of ecological, developmental and evolutionary events that give raise to systematic differences between island and mainland populations, jointly known as 'island syndrome' or 'insular syndrome' (Blondel, 
1986; Libois et al., 1993; Adler and Levins, 1994). Island and mainland populations of several animal species, including terrestrial small mammals differ in morphological, behavioural, metabolic, and life history traits (Tamarin, 1978; Libois et al., 1993; Adler and Levins, 1994; Magnanou et al., 2005; Cornette et al., 2012; Moncunill-Solé et al., 2016; Abramov et al., 2017). Moreover, because island populations usually originate from a small number of founder individuals, they often harbour lower levels of genetic diversity than mainland populations (Frankham, 1997).

In shrews (Soricomorpha, Soricidae), insularity has been related to increased body size (White and Searle, 2007a), rapid morphofunctional changes (Cornette et al., 2012), and metabolic alterations (Magnanou et al., 2005). In addition, it has also been observed that the island populations have lower genetic diversity than the mainland ones, and that genetic diversity decreases with the size of both the island and the population (White and Searle, 2007b). Shrews are generalist carnivores that mainly feed on soil invertebrates, which is a highly variable food resource among the different terrestrial habitats. They are amongst the smallest mammals, and as such, they have high basal metabolic rate and often have to deal with severe constraints in order to maintain their thermal and energetic balance (Magnanou et al., 2005). As a result, shrews have a reduced energetic autonomy and they need to be continuously foraging (Genoud, 1988). Besides, they are territorial and aggressive animals that fight and bite with each other in competitive interactions, either with individuals of the same species or of other species (Churchfield, 1990; Cornette et al., 2015; McDevitt et al., 2014; Montgomery et al., 2015).

Previous studies have shown that the mandible of shrews is a suitable model for demonstrating differences in ecology and selective regimes among populations (Young et al.,2010). Because the mandible plays a central role in the capture and processing of the food, and also in competitive interactions, differences in mandibular form among populations may highlight functional advantages. The functional capacity of the mandible is usually assessed by the mechanical potential (MP), which may reflect selection on bite performance (Young et al., 2007; Cornette et al., 2012, 2015). The ability to bite hard allows an animal to capture and reduce food, and it has direct consequences on diet (Cornette et al., 2015). Moreover, biting is relevant in a competitive context and may determine the outcome of direct aggressive interactions (Downes and Bauwens, 2002). A high bite force capacity, thus, likely offers a selective advantage to individuals by having both a wider range of available food resources and superiority in inter and intraspecific interactions (Christiansen and Wroe, 2007; Anderson et al., 2008). As explained above, insularity can produce a decrease in species richness and an increase in population density (Adler and Levins, 1994). While the decrease in species richness might affect the availability and/ or quality of prey for the shrews, the decrease in the diversity of competitors as well as the increase of population density might affect their competitive interactions. Therefore, the selective pressures acting on MP of the mandible in island populations of shrews might differ from the mainland ones. Since, the MP is strongly dependent on mandible morphology, changes in the form of the mandible might be expected in island populations of shrews (Young et al., 2007; Cornette et al., 2012, 2015).

The greater white-toothed shrew Crocidura russula (Hermann, 1780) is a relatively sedentary small carnivore with high feeding requirements that is able to eat a great variety of preys (Churchfield, 1990). This crocidurine is widespread across Western Europe (except Italy) and Northern Maghreb, including some Atlantic and Mediterranean islands (Cornette et al., 2012 and references therein). Moreover, C. russula has been recently reported as a highly invasive species with rapid adaptation to new territories and high potential to displace other shrews, disrupting autochthonous small mammals communities (McDevitt et al., 2014; Montgomery et al., 2015; Gargan et al., 2016). Mainland and island populations of $C$. russula have been considered largely undifferentiated; nevertheless, recent studies do have detected metabolic and morphological differences (Magnanou et al., 2005; Cornette et al., 2012, 2015). Specifically, while morphological studies have analysed mandible form in populations of $C$. russula from Atlantic islands (Cornette et al., 2012, 2015; Gargan et al., 2016), similar information regarding Mediterranean populations is very scarce. Since evolutionary causes prompting morphological change in insular populations can differ depending on ecological and geographic features of the islands (Lomolino et al., 2012), the study of populations of C. russula from Mediterranean islands can bring important information of this widely distributed and highly invasive small mammal species (McDevitt et al., 2014; Montgomery et al., 2015; Gargan et al., 2016). In this context, the general aim of this study is to gain insight into the evolutionary and ecological 


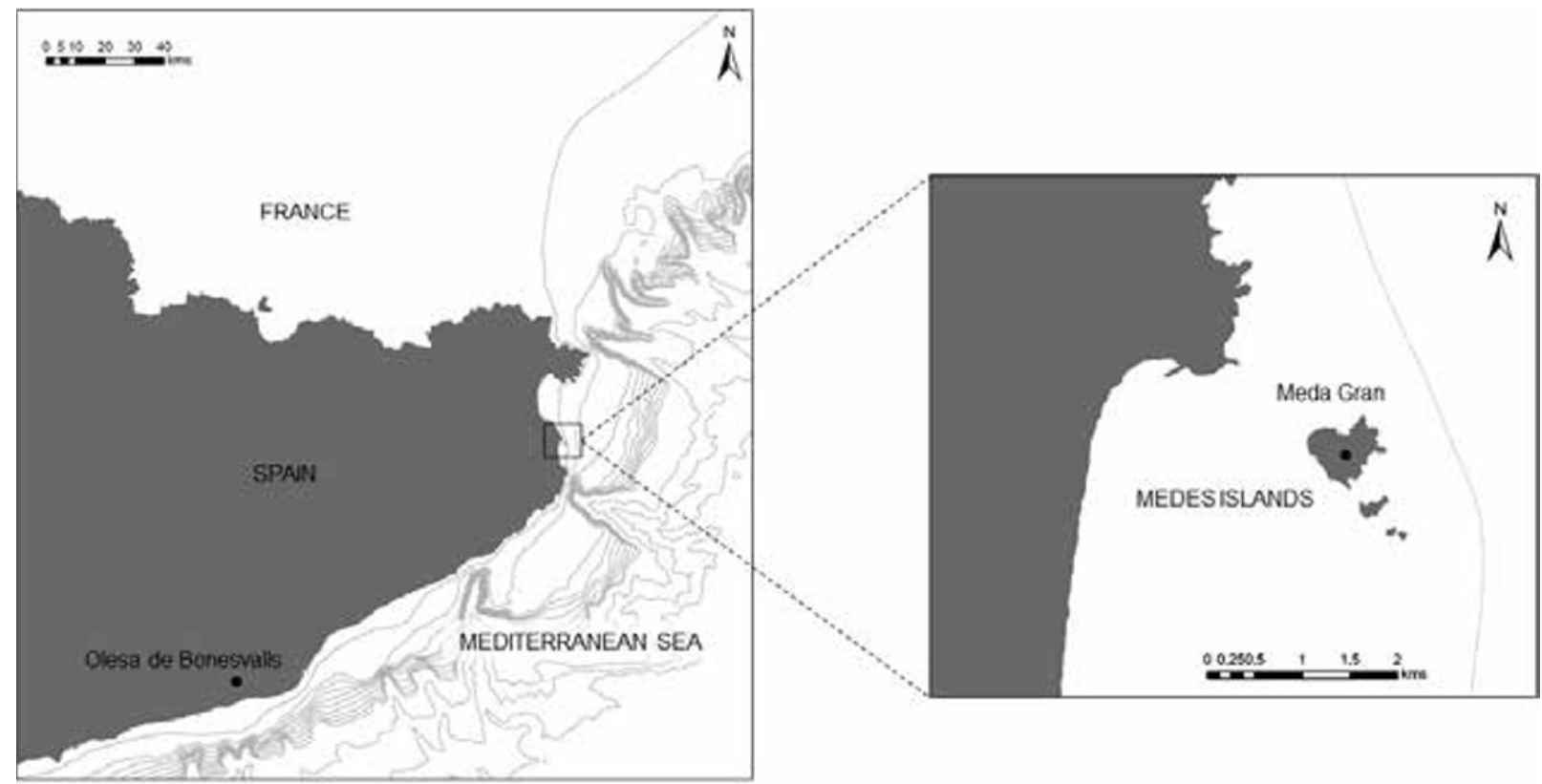

Figure 1. Location of the sampled populations.

processes involved in morphological differentiation of island populations of $C$. russula. To accomplish it, we analyse changes in body size, mandible form, and mechanical potential in a population of $C$. russula from the Medes Islands archipelago, a group of small islands and islets located in front of the coast from l'Estartit (Girona Province, NE Iberian Peninsula). We compare this population with mainland animals with three specific and complementary objectives. First, we evaluate if shrews from Medes Islands suffer changes in body size and body condition index, as it has been previously detected in insular populations of several species of small mammals. Second, we analyse the form of the mandible to uncover if mandible size and shape differ with regard to mainland shrews. Third, we study changes in the mechanical potential and its association with both size and shape variation to uncover if changes in mandible form have functional consequences.

\section{Material and Methods}

Study sites and sample collection

The mainland sample composed of 25 adult shrews (18 males, 7 females) was collected in Olesa de Bonesvalls (Natural Park of Garraf, NE Iberian Peninsula). The island sample composed of 28 adult shrews (12 males, 16 females) was obtained in la Meda Gran (Medes Islands and Baix Ter Natural Park, NE Iberian Peninsula; Fig. 1). Both capture sites are semi-arid Mediterranean areas with similar ecological conditions regarding altitude, latitude, habitat composition, and landscape. Moreover, both sites are included in protected areas and are not affected by relevant anthropogenic pollution sources (SánchezChardi and Nadal, 2007; Sánchez-Chardi et al., 2007; Sánchez-Chardi and López-Fuster, 2009). These specimens have been previously used in ecological and ecotoxicological studies (Sánchez-Chardi and Nadal, 2007; Sánchez-Chardi et al., 2009; SánchezChardi and López-Fuster, 2009; Sánchez-Chardi et al., 2013).

\section{Body mass, body length and body condition}

Body mass (BM, in $\mathrm{g}$ ) and body length (BL, in $\mathrm{mm}$ ) of each animal were measured immediately after its capture and euthanization; for pregnant females, the mass of the embryos was subtracted from the female body mass. For BM and BL separately, differences between sites, sexes, and their interaction were evaluated using type III sums of squares (SS) factorial ANOVA models. If the interaction was not significant, it was dropped from the model and type II SS ANOVA models were applied. Given that a tendency of differences in BM between sexes in the 
Table 1. Anatomical definitions of the collected landmarks showed in Fig. 2.

\begin{tabular}{ll}
\hline Number & Landmark description \\
\hline 1 & Dorsal-most point of the coronoid process \\
2 & Dorsal-most point of the articular condyle \\
3 & Posterior-most point of the articular condyle \\
4 & Ventral-most point of the articular condyle \\
5 & Posterior-most point of the angular process \\
6 & $\begin{array}{l}\text { Inflexion point between the angular process and the } \\
\text { mandibular body }\end{array}$ \\
7 & $\begin{array}{l}\text { Inflexion point of the mandibular body } \\
8\end{array}$ \\
9 & $\begin{array}{l}\text { Anterior-most point of the mandible at the base of } \\
\text { the incisor }\end{array}$ \\
10 & Posterior alveolar margin of canine \\
11 & Posterior alveolar margin of M1 \\
12 & Anterior-most corner of the coronoid fossa \\
\hline
\end{tabular}

population of Medes Islands was detected, we also conducted an exploratory t-test between sexes in this site. To calculate the body condition index (BCI) of an individual, it is necessary to know beforehand whether differences in the BM-BL linear relationship exist between populations and/or sexes (see García-Berthou, 2001). We explored these differences using a model selection procedure. We used the function dredge, as implemented in the package MuMIn (Barton, 2016), to compute and rank all possible linear models explaining the cube root of BM. Since there is a cubic relationship between $\mathrm{BM}$ and $\mathrm{BL}$, we used the cube root of BM to improve the linear relationship among the continuous variables. The full model included $\mathrm{BL}$ as a covariate, both site and sex as factors, and all possible interactions. We ranked models according to Akaike information criterion (AIC), Bayesian information criterion (BIC) and second-order AIC (AICc). Once the best model was identified, we obtained p-values for the most important variables or terms using type III SS ANOVAs - to test for interactions - or type II SS ANOVAs for covariates or factors - if interactions were not significant and were dropped from the model. Given that the BM-BL relationship differed between populations (see Results), we used as individual BCIs the residuals of the regression, for each population separately, of BM on BL. All these analyses were made using R (R Core Team, 2015).

\section{Acquisition of mandible form data}

The form of the mandibles was analysed by geometric morphometric techniques implemented in MorphoJ (Klingenberg, 2011). The right and left mandibles of each specimen were separated at the mandibular symphysis and photographed in lingual view with a digital camera (Canon Power shot, 1.2 million pixels). Twelve two-dimensional landmarks (LM) were digitized in each mandible (Table 1 and Fig. 2A) using TpsDig 2.16 (Rohlf, 2010). In order to avoid an additional source of bias, the images of the left mandibles were rotated horizontally $180^{\circ}$ prior to digitization (White and Searle, 2008). Measurement error is an important source of variation affecting morphometric data (Bailey and Byrnes, 1990). Consequently, to assess measurement error, landmarks were digitized twice by the same person (MGP). The form defined by each set of LMs was decomposed into size and shape components, which were analysed separately. Centroid size (CS, the square root of the sum of squared distances of each landmark to the centroid of the landmarks configuration) was used as a measure of size (Dryden and Mardia, 1998). Shape information was extracted from the landmark coordinates with a full Procrustes fit and the orthogonal projection of data to the tangent space (Dryden and Mardia, 1998). Coordinates resulting from the Procrustes fit (Procrustes coordinates) were used as shape variables. Variations in shape and size were decomposed into symmetric and asymmetric components, i.e. the variation among individuals in terms of the averages of the landmark configurations of both sides, and the variation within individuals regarding the differences in landmark configurations of the two sides, respectively (Klingenberg et al., 2002). The symmetric component was used to study size and shape variation among populations and sexes.

\section{Analyses of mandible size and shape}

First, in order to estimate the sources of variance in mandible size, a two-factor ANOVA was conducted, with side and individual as fixed and random factors, respectively (Palmer and Strobeck, 1986). In this ANOVA, the factor individual accounts for individual variation and the factor side for directional asymmetry (DA). The interaction between both factors 
estimates the measure of fluctuating asymmetry (FA), whereas residual variance component between replicates quantifies measurement error. To assess the geographical effect and the sexual dimorphism on mandible size, a factorial ANOVA with sex and site as categorical predictors and symmetric CS (average of left and right sides) as dependent variable was conducted. Since significant differences in body mass and body length were observed between both sites (see results), an ANCOVA with body mass as co-variable was used in order to control for overall body size.

To estimate the sources of variance in mandible shape, a Procrustes ANOVA was performed, which is equivalent to the ANOVA developed by Palmer and Strobeck (1986) but adapted for the study of shape (Klingenberg and McIntyre, 1998). Allometry, i.e. size-dependent shape changes, is a potential factor affecting shape variation (Klingenberg, 2009). To assess allometric shape changes of the mandible, a pooled within-populations multivariate regression of the symmetric component of shape (i.e. the averages of the Procrustes coordinates of the left and right sides) on symmetric CS was performed. Statistical significance was tested using permutation tests with 10000 iterations under the null hypothesis of no allometric relationship (Monteiro, 1999). Since a

A
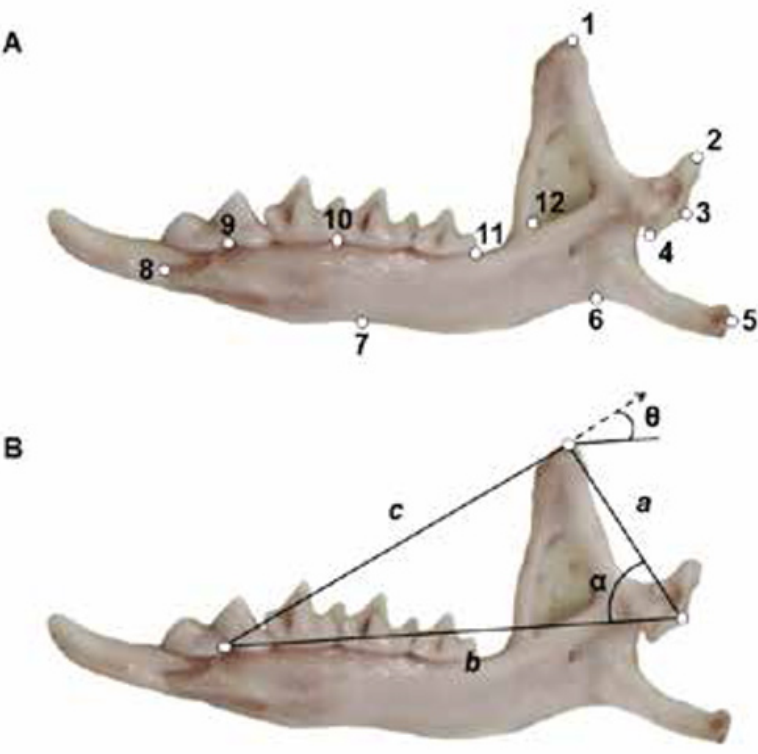

$5 \mathrm{~mm}$

Figure 2. Lingual view of the right hemimandible with the location of the 12 recorded landmarks (A), and the measures and angles used to calculate the mechanical potential (B). significant dependence of shape on size was not found (see Mandible Size and Shape section), subsequent analyses were based on raw data (i.e. symmetric component of shape not corrected by size). Shape differences among sites and sexes were assessed by a factorial MANOVA using the symmetric component of shape as dependent variables. Patterns of shape variation were explored with a principal component analysis (PCA). A canonical variate analysis (CVAs) and a discriminant function analysis (DFAs) were conducted to examine the shape features that best distinguished between sites. The Mahalanobis distance between the centroids of both sites was calculated and the statistical differences between group means were assessed with a T-square test in which $p$-values were obtained using a permutation procedure with 10000 iterations.

\section{Mechanical potential}

To evaluate the performance of the mandible we calculated the mechanical potential (MP), which provides an approximation of the force that can be generated by the temporalis muscle during biting (Cornette et al., 2015). The MP of each individual was calculated by applying a biomechanical model in which the mandible is considered a simple lever (Young et al., 2007; Cornette et al., 2012, 2015). In this model, the MP was calculated as:

$\mathrm{MP}=a / b^{*} \cos (\theta)$

where $a$ is the distance from the tip of the coronoid process (landmark 1) to the most ventral point of the condylar process (landmark 4$), b$ is the distance from this point to the posterior alveolar margin of canine (landmark 9), and $\theta$ is the force angle, which can be calculated by the following formula:

$$
\theta=90-\alpha
$$

In this formula $\alpha$ is the gape angle, i.e. the angle between the distances $a$ and $b$, and can be obtained by the formula:

$$
\alpha=\arccos \left[\left(a^{2}+b^{2}-c^{2}\right) 2 a b\right]
$$

where $c$ is the distance from the tip of the coronoid process (landmark 1) to the posterior alveolar margin of canine (landmark 9; Fig. 2B).

To evaluate the influence of size on MP, the linear regression of the MP on the symmetric CS was performed. Statistical significance was tested using permutation tests with 10000 iterations under the null hypothesis of no relationship. Since no dependence of the MP onto CS was detected, differences on MP among sites and sexes were tested with a factorial 
ANOVA using type III SS. If the interaction was not significant, it was dropped from the model and type II SS was used. We also performed a multivariate regression of the symmetric component of shape on MP to quantify and visualize the shape changes associated to changes in the MP. In order to assess to which degree shape differences among sites were associated to changes in MP, we performed a linear regression of the first canonical variable (CV1) on MP. The statistical significance in both cases was assessed using a permutation tests with 10000 iterations under the null hypothesis of no relationship.

Previously to perform further analyses, all variables were tested for normality and homogeneity of variances employing the Shapiro-Wilk and the Levene test, respectively.

\section{Results}

Body mass, body length and body condition index

The Shapiro-Wilk and the Levene tests indicated that all variables followed a normal distribution and showed homogeneity of variances among sites and sexes. Measures of BL and BM differed between populations. Insular shrews were longer $\left(x_{\text {Medes }}^{-}=74.67 \pm 3.40\right.$; $\left.x_{\text {Garraf }}^{-}=69.91 \pm 3.23 ; F_{1,46}=31.14, p<0.001\right)$, and heavier $\left(x_{\text {Medes }}^{-}=8.40 \pm 0.75 ; x_{\text {Garraf }}^{-}=7.30 \pm 0.72 ; F_{1,46}\right.$ $=29.83, p<0.001)$. Differences between sexes were not found in $\mathrm{BM}\left(x_{\text {males }}^{-}=7.82 \pm 0.98 ; x_{\text {females }}^{-}=7.86\right.$ $\pm 0.83 ; F_{1,46}=2.15, p=0.15$ ), but males were slightly longer than females $\left(x_{\text {males }}^{-}=72.39 \pm 4.29 ; x_{\text {females }}^{-}=\right.$ $\left.72.04 \pm 3.84 ; F_{1,46}=4.29, p=0.04\right)$. For both variables, the site and sex interaction was not significant (BL: $F_{1,45}$ $=0.04, p=0.84$; BM: $\left.F_{1,45}=1.96, p=0.17\right)$. Although the interaction between sex and site was not significant for BM, a further exploration of the interaction plot and marginal means showed that, for the insular shrews, there was a trend for the males to be heavier than the females $\left(x_{\text {males }}^{-}=8.75 \pm 0.67 ; x_{\text {females }}^{-}=8.14 \pm 0.72 ; t_{22}=\right.$ $2.09 ; p=0.048$; Fig. S1).

Regarding the BM-BL relationship, the best model according to all ranking criteria included only BL and site (Table S1). Insular shrews had higher BM than continental specimens when accounting for BL $\left(F_{1,46}\right.$ $=10.50, p<0.01)$. However, although the site effect was important according to all methods, the inclusion of BL was marginally necessary according to ANOVA tests $\left(F_{1,46}=3.99, p=0.05\right)$. The second best model according to AIC includes the interaction between

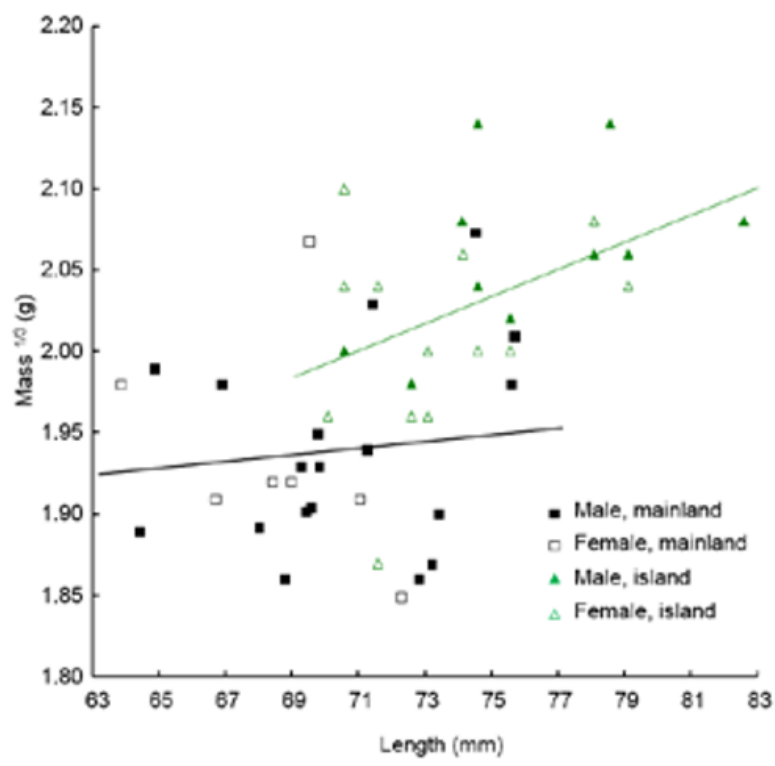

Figure 3. The relationship between body length (BL, in $\mathrm{mm}$ ) and the cube root of body mass (BM, in $\mathrm{g}$ ) for the two sampled populations.

site and BL (Table S1), suggesting that insular shrews also tended to enhance its BM more rapidly when $\mathrm{BL}$ increases than mainland shrews (Fig. 3). However, ANOVA results did not support this difference $\left(F_{1,45}=\right.$ $1.47, p=0.23$ ).

\section{Mandible size and shape}

The two-factor ANOVA performed on CS revealed that variation resulting from measurement error was negligible since it was significantly exceeded by variation in FA (Table 2). Significant differences in mandible size were detected between sexes and sites (sex: $F_{1,49}=8.4, p<0.01$; site: $F_{1,49}=8.5, p<0.01$; interaction: $F_{1,49}=0.8, p=0.38$ ). Males had bigger mandibles than females, and insular animals had bigger mandibles than continental ones (Fig. 4). However, because the size of the body parts depends on the total size of the animal, the differences detected on mandible CS could be due to changes in body size. This fact was confirmed by the ANCOVA, which showed that while differences in mandible size between sites are exclusively due to changes in body size, there is sexual dimorphism for the size of the mandible $\left(\mathrm{BM}: F_{1,45}=\right.$ 23.94, $p<0.01$; site: $F_{1,45}=0.08, p=0.78$; sex: $F_{1,45}=$ $5.30, p<0.05)$.

The Procrustes ANOVA revealed that measurement error is not a serious concern for the study of shape, 
since variation in FA significantly exceeded variation resulting from measurementerror (Table 3). A significant dependence of the symmetric shape component onto symmetric CS was not detected. Site but not sex had an effect on the mandible shape (sex: $F_{30,20}=0.74, p$ $=0.76$; site: $F_{30,20}=7.91, p<0.01$; interaction: $F_{30,20}$ $=1.86, p=0.06$ ). Differences among populations were further corroborated by PCA. The first two PCs, which together explained $49.1 \%$ of the variance, clearly separated the two populations, mainly along the first principal component (Fig. 5). Shape changes associated to these first two PCs were concentrated in the proximal part of the mandible, mainly affecting the coronoid, the condylar, and the angular processes (Fig. 5). The CVA and DFA also revealed the existence of shape differences between the two populations of shrews, the Mahalanobis distance between them being statistically significant $(\mathrm{MD}=4.53, p<0.01)$. Shape diverged between populations, with insular specimens having a higher coronoid process, a broader condyloid process, and a more downward projected angular process than continental ones.

\section{Mechanical potential}

The linear regression indicated that the MP did not depend on CS $(p=0.92)$. The ANOVA revealed significant differences in MP between sites but not between sexes (site: $F_{1,49}=18.91, p<0.001$; sex: $F_{1,49}$ $=0.01, p=0.98)$, island populations having higher MP values than mainland ones (Fig. 6), and with a

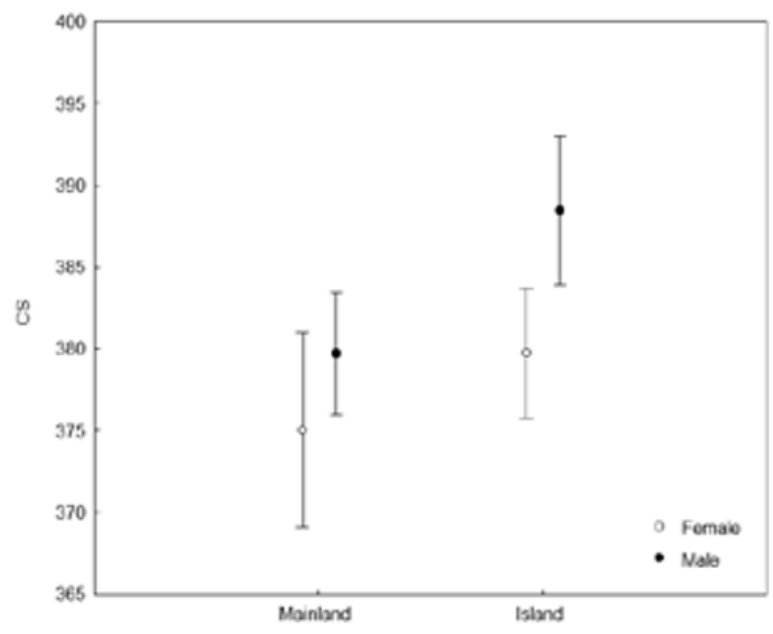

Figure 4. Mean centroid size of the mandible in males and females from the two analysed populations. Vertical bars denote 0.95 confidence intervals.
Table 2. Two-way ANOVA for centroid size conducted on the whole sample.

\begin{tabular}{lrrrrr}
\hline Effect & \multicolumn{1}{c}{ SS } & \multicolumn{1}{c}{ MS } & df & \multicolumn{1}{c}{$F$} & \multicolumn{1}{c}{$p$} \\
\hline Individual & 16009.67 & 307.88 & 52 & 30.21 & $<0.001$ \\
Side & 17.45 & 17.45 & 1 & 1.71 & 0.196 \\
Ind $\times$ Side & 529.93 & 10.19 & 52 & 4.85 & $<0.001$ \\
Error & 222.80 & 2.10 & 106 & & \\
\hline
\end{tabular}

* SS: sum of squares; MS: mean squares; df: degrees of freedom; F: F statistic; $p$ : $p$-value.

non-significant interaction among both factors $\left(F_{1,49}\right.$ $=3.75, p=0.06)$. A clear association between MP and mandible shape was detected $(p<0.01)$, with changes in MP explaining almost a $16.0 \%$ of variation in mandible shape (Fig. 7). Shape changes associated to MP mainly affected the mandible processes. These changes consisted in an increase of the height and a frontward projection of the coronoid process, an expansion of the condylar process, and a slight enlargement and downward projection of the angular process (Fig. 7). The linear regression of CV1 on MP indicated that $35.3 \%$ of shape differences between sites were explained by changes in MP $(p<0.01)$.

\section{Discussion}

Patterns of size change on islands have received considerable attention (e.g. Michaux et al., 2002; Lomolino, 2005; Raia and Meiri, 2011). It has been observed that species of big mammals tend to become smaller and species of small mammals tend to become larger on islands, a trend that has been called 'island rule' (Van Valen, 1973; Michaux et al., 2002; Lomolino, 2005). In shrews, increased body size has been reported in Sorex araneus (White and Searle, 2007a; Frafjord, 2008), C. suaveolens (Magnanou et al., 2005), and $C$. russula (Cornette et al., 2012) from Atlantic islands. Our results are consistent with these findings, with insular shrews from a Mediterranean island being $16 \%$ heavier and $10 \%$ longer than mainland specimens (according to mean values). Data of previous studies support that changes in body size are positively related to the distance between the island and mainland and negatively related to island size (White and Searle, 2007a; Millien, 2011). Although Meda Gran is located only $1.5 \mathrm{~km}$ apart from the coast, and therefore changes in body size would not be expected, it is a very small island $\left(0.18 \mathrm{~km}^{2}\right)$, which could explain the considerable change in body size detected in shrews in the present 

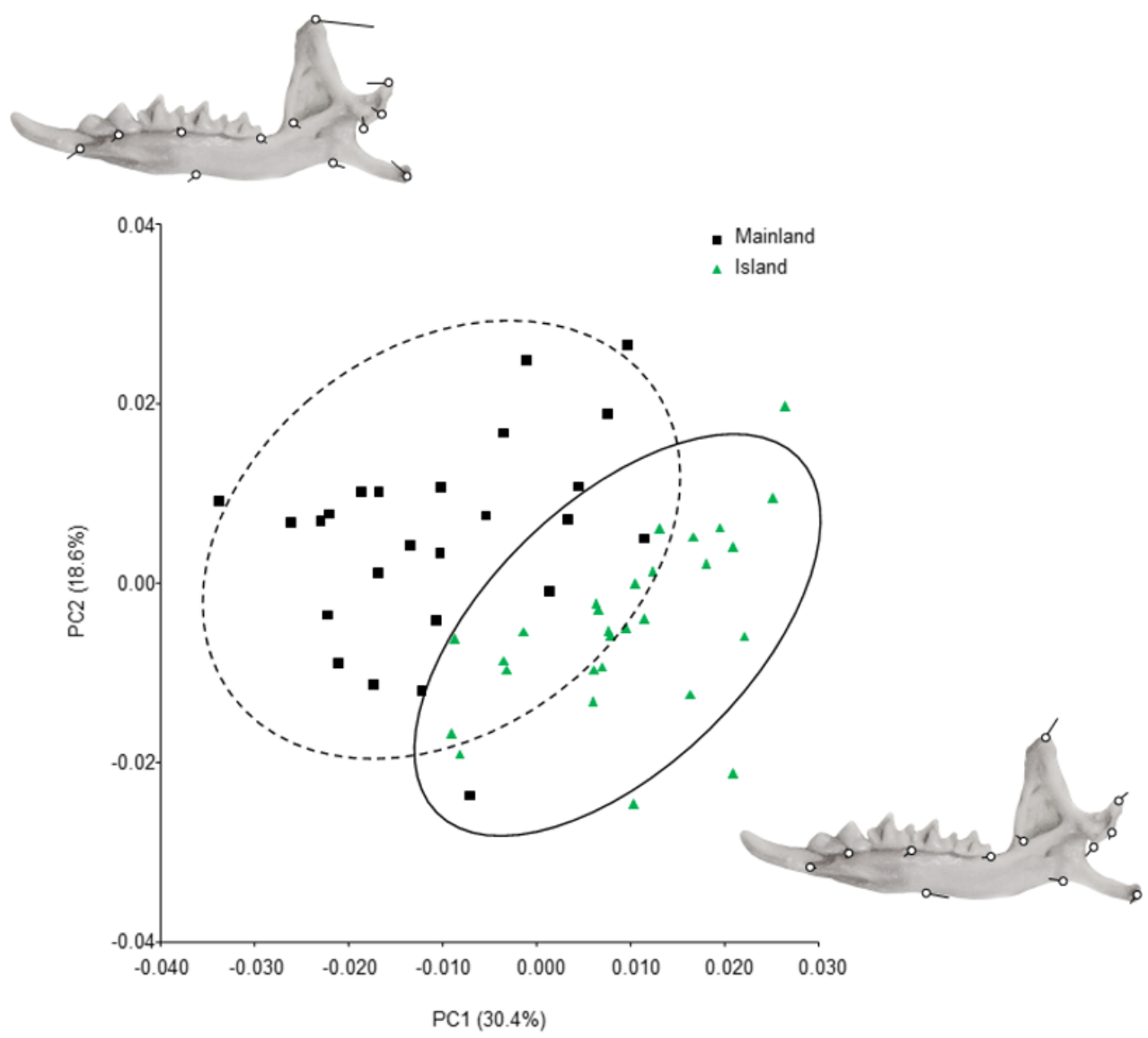

Figure 5. Plot of the individual values of the two first principal components (PC1 and PC2). The percentage of shape changes explained by each PC is indicated within parentheses. Shape changes associated to $\mathrm{PC} 1$ and $\mathrm{PC} 2$ are depicted in each axis; dots correspond to the consensus shape and the vectors to the shape change that produce a displacement of 0.1 units in the positive direction of the PC.

study. Size increase in island populations of small mammals might result from environmental variation and/or genetic changes prompted by genetic drift and/ or natural selection. However, the recurrent pattern of size increase in insular populations of soricids suggests that non-random processes are responsible for this differentiation. Therefore, size increase in insular populations is probably due to genetic changes occurring as a rapid response to a new selective regime. Thus, size increase in insular populations of small mammals has been recently associated to immigrant selection on remote islands, thermoregulation and endurance on islands from temperate latitudes with cold and highly seasonal climates, and ecological release on small islands lacking mammalian predators and competitors (Lomolino et al., 2012). Meda Gran is a small island only inhabited by three mammal species, Oryctolagus cuniculus, Mus musculus, and C. russula (Ros et al., 1984). Therefore, without mammalian predators or competitors, ecological release seems to

Table 3. Procrustes ANOVA for shape conducted on the whole sample.

\begin{tabular}{lccccccc}
\hline Effect & $\mathrm{SS}$ & $\mathrm{MS}$ & $\mathrm{df}$ & $F$ & $p$ & Pillai's tr & $p$ \\
\hline Individual & 0.149 & $1.43 \times 10-4$ & 1040 & 5.32 & $<0.001$ & 14.89 & 0.66 \\
Side & 0.003 & $1.35 \times 10-4$ & 20 & 5.03 & $<0.001$ & $<.001$ & 0.001 \\
Ind $\times$ Side & 0.028 & $2.69 \times 10-5$ & 1040 & 2.87 & $<0.001$ & $<0.001$ \\
Error & 0.0199 & $9.38 \times 10-6$ & 2120 & & & \\
\hline
\end{tabular}

* SS: sum of squares; MS: mean squares; df: degrees of freedom; F: F statistic; $p$ : $p$-value. 
be the most plausible explanation for size increase in specimens of $C$. russula from Meda Gran. Ecological release has been also pointed out as the main factor driving size increase in insular populations of other species (Ventura and López-Fuster, 2000; Herczeq et al., 2009; Russell et al., 2011). Our results also suggest the existence of a slight sexual size dimorphism in BLin both populations of $C$. russula, with males longer than females. Moreover, the analyses showed a trend for the males to be heavier than the females only in the insular population (Fig.3 and Fig. S1). Sexual size dimorphism has been previously detected in this species, and it has been attributed to a stronger directional selection for increased body size in males than in females associated to territorial behaviour (Boutellier-Reuter and Perrin, 2005). Although in $C$. russula both sexes are known to participate actively in territory defence (Cantoni and Vogel, 1989), males play the main role (BoutellierReuter and Perrin, 2005).

Mandible form, both size and shape, also differed between the island and the mainland populations. Differences in mandible size were detected between populations and sexes, with males having bigger mandibles than females, and the insular individuals bigger than the mainland ones. However, while differences in mandible size between populations were due to the increase in body size, differences between sexes were not. This result indicates that sexual dimorphism also exists in mandible size of the analysed sample of $C$. russula. In addition, this dimorphism seemed to be more evident in island than in mainland

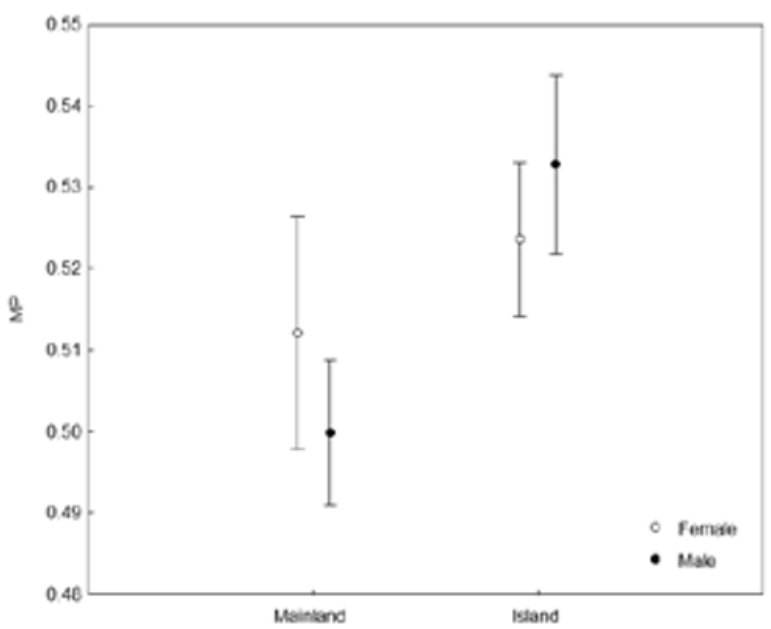

Figure 6. Mean mechanical potential of the mandible in males and females from the two analysed populations. Vertical bars denote 0.95 confidence intervals. populations. While this seems to support the hypothesis of the increase in the number of agonistic encounters prompted by an increase in population density (individuals with bigger mandibles perform better in combats), we did not detect an association between mandible size and MP. Significant differences in mandible shape were detected between populations but not between sexes. Shape changes were mainly located in the posterior part of the mandible, as previously described by Cornette et al. (2012). In fact, the shape changes detected in Meda Gran are coincident to those observed in two small Atlantic islands, Molène and Groix (Cornette et al., 2012).The observed differences in mandible shape could be the result of several genetic and epigenetic factors. Thus, shape differences could be of environmental origin, being, for example, the result of a plastic response of the bone to a change in the consistency of the food (Young et al., 2010). They could also result from genetic changes, either stochastic, such as founder effect, or selective. The fact that the observed mandible shape changes are tightly linked to an increase in MP, suggests that they are not stochastic, but adaptive. Similar results were obtained in a previous study analysing mandible variation in several Atlantic islands (Cornette et al., 2012). In this study, it was suggested that changes in the mechanical potential were due to a shift in the trophic niches of insular populations (Cornette et al., 2012). Although we do not have precise data about diet composition in both populations, a previous study suggests that the diet of $C$. russula from Meda Gran might be similar to mainland populations based on the similarity of insect communities between both regions (Ros et al., 1984). If this were the case, a shift in the trophic niche would not explain changes in mandible shape and the resulting increase in MP. Because of the central role of the mandible in competitive interactions, changes in the mechanical potential could also obey to selective pressures in territorial behaviour. For instance, it has been observed that common shrews with longer and broader coronoid and condylar processes have a greater mechanical potential and perform better in combats (Hanski et al., 1991; White and Searle, 2009). This explanation would also fit with ecological release, since an increase in population density would imply a higher number of intraspecific competitive encounters.

In the light of these results, we conclude that insular specimens of the greater white-toothed shrew C. russula differ from continental ones in several morphological traits. Overall, shrews inhabiting Meda Gran were longer, heavier, and showed a different 


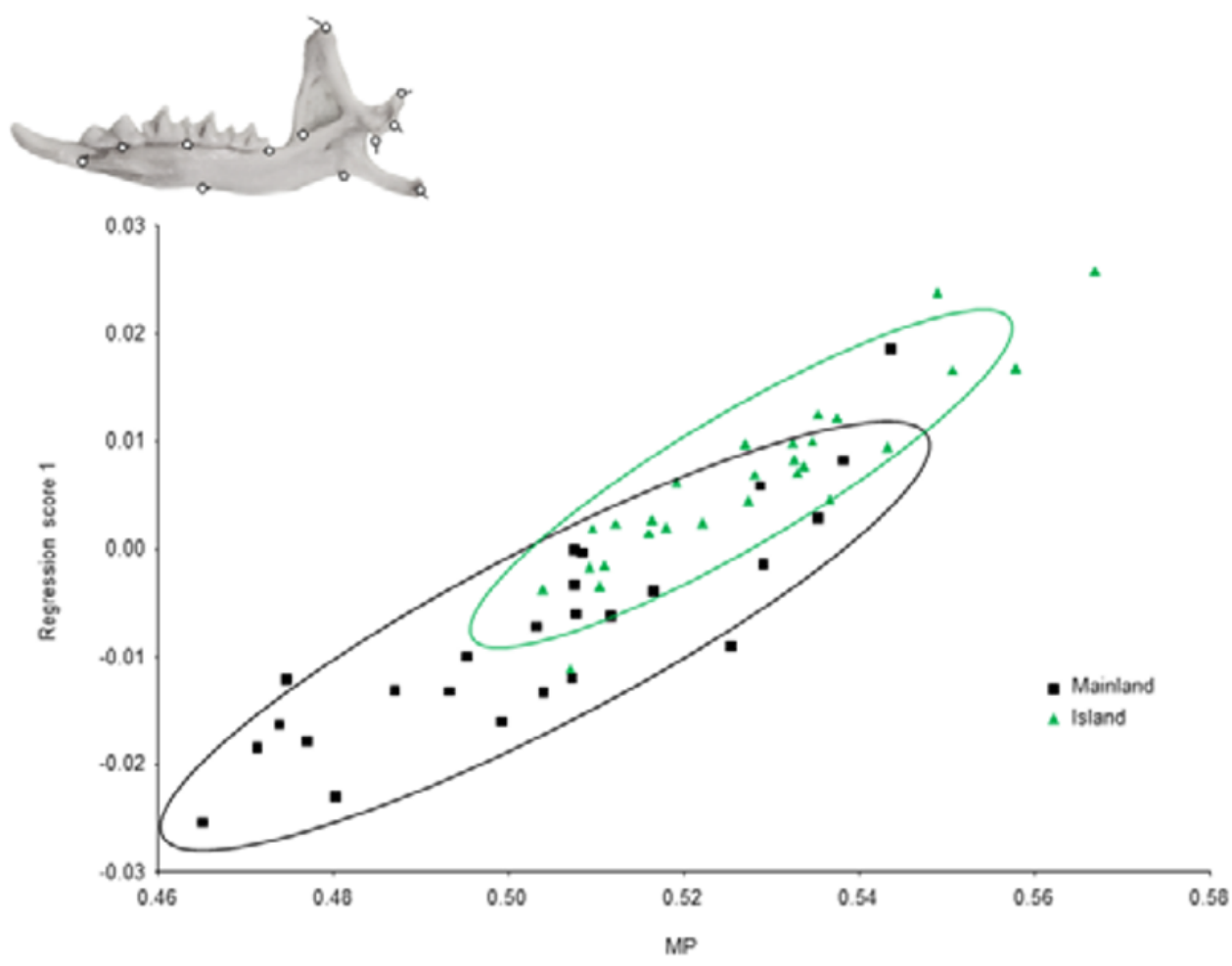

Figure 7. Plot of the multivariate regression of shape against mechanical potential. Shape changes associated to mechanical potential are depicted as vector displacements; dots correspond to the consensus shape and the vectors to the shape change from the mandible with the smallest MP value to the mandible with the highest MP value. mandible shape with higher MP when compared with continental specimens. They also showed better physical condition than mainland conspecifics. Presumably, overall changes in phenotypic traits could be the result of two main interrelated factors occurring in ecological release, the decrease of predation pressure and interspecific competition and the consequent increase of intraspecific competition. While the increase in size and body condition in island shrews would result from reduced predation and interspecific competition, the changes in mandible shape and the increase of MP and sexual dimorphism would arise as a response to the greater intraspecific competition driven by increased population density. Studies analysing predation pressure, diet composition, population density and competition are needed to determine the specific role of ecological release in morphological changes in island populations of C.russula.

\section{Acknowledgements}

The authors thank Dr. Juan José Egozcue (Universitat Politècnica de Catalunya) and Ms. Núria Garriga (Universitat de Barcelona) for solving some methodological issues, and Dr. Anna Soler for her help with the map figure. Two anonymous reviewers made valuable comments for which we thank them. This study was partially supported by the Generalitat de Catalunya (Projects 2002ACOM00012 and 2009SGR403) and the Museu de Gavà.

\section{References}

Abramov SA, Lopatina NV, Litvinov YN. 2017. Cranial size and shape variation in isolated populations of the Olkhon mountain vole (Alticola olchonensis Litvinov, 1960). Zoology 123: 91-100.

Adler GH, Levins R. 1994. The island syndrome in rodent populations. The Quarterly Review of Biology 69: 473-90.

Anderson RA, McBrayer LD, Herrel A. 2008. Bite force in vertebrates: opportunities and caveats for use of a nonpareil whole animal performance measure. Biological Journal of the Linnean Society 93: 709-720.

Bailey RC, Byrnes J. 1990. A new, old method for assessing measurement error in both univariate and multivariate morphometric studies. Systematic Zoology 39: 124-130.

Bartoń K. 2016. MuMIn: Multi-Model Inference. R package version 1.15.6: https://CRAN.R-project.org/ package $=$ MuMIn

Blondel J. 1986. Biogéographie évolutive. Paris: Masson.

Boutellier-Reuter C, Perrin N. 2005. Sex-specific selective pressures on body mass in the greater white-toothed shrew, Crocidura russula. Journal of Evolutionary Biology 18: 290300 .

Cantoni D, Vogel P. 1989. Social organization and mating system of free-ranging, greater white-toothed shrews, Crocidura russula. Animal Behaviour 38: 205-214. 
Christiansen P, Wroe S. 2007. Bite forces and evolutionary adaptations to feeding ecology in carnivores. Ecology 88: 347-358.

Cornette R, Herrel R, Cosson JF, Poitevin F, Baylac M. 2012. Rapid morpho-functional changes among insular populations of the greater white-toothed shrew. Biological Journal of the Linnean Society 107: 322-331.

Cornette R, Tresset A, Houssin C, Pascal M, Herrel A. 2015. Does bite force provide a competitive advantage in shrews? The case of the greater white-toothed shrew. Biological Journal of the Linnean Society 114: 795-807.

Churchfield S. 1990. The Natural History of Shrews. London: Christopher Helm.

Downes S, Bauwens D. 2002. An experimental demonstration of direct behavioural interference in two Mediterranean lacertid lizard species. Animal Behaviour 63: 1037-1046.

Dryden IL, Mardia KV. 1998. Statistical shape analysis. Chichester: Wiley.

Frafjord K. 2008. Can environmental factors explain size variation in the common shrew (Sorex araneus)? Mammalian Biology 73: 415-422.

Frankham R. 1997. Do island populations have less genetic variation than mainland populations? Heredity 78: 311-317.

Gargan LM, Cornette R, Yearsley JM, Montgomery WI, Paupério J, Alves PC, Butler F, Pascal M, Tresset A, Herrel A, Lusby J, Tosh DG, Searle JB, McDevitt AD. 2016. Molecular and morphological insights into the origin of the invasive greater white-toothed shrew (Crocidura russula) in Ireland. Biological Invasions 18: 857-871.

García-Berthou E. 2001. On the misuse of residuals in ecology: testing regression residuals vs. the analysis of covariance. Journal of Animal Ecology 70: 708-711.

Genoud M. 1988. Energetic strategies of shrews: ecological constraints and evolutionary implications. Mammal Review 18: 173-193.

Hanski I, Peltonen A, Kaski L. 1991. Natal dispersal and social dominance in the Common Shrew Sorex araneus. Oikos 62: 48-58.

Herczeq G, Gonda A, Merilä J. 2009. Evolution of gigantism in nine-spined sticklebacks. Evolution 63: 3190-3200.

Klingenberg CP. 2009. Morphometric integration and modularity in configurations of landmarks: tools for evaluating a priori hypotheses. Evolution and Development 11: 405-421.

Klingenberg CP. 2011. MorphoJ: an integrated software package for geometric morphometrics. Molecular Ecology Research 11: 353-357.

Klingenberg CP, Barluenga M, Meyer A. 2002. Shape analysis of symmetric structures: quantifying variation among individuals and asymmetry. Evolution 56: 1909-1920.

Klingenberg CP, McIntyre GS. 1998. Geometric morphometrics of developmental instability: analyzing patterns of fluctuating asymmetry with Procrustes methods. Evolution 52: 1363-1375.

Libois R, Fons R, Bordenave D. 1993. Mediterranean small mammals and insular syndrome: biometrical study of the long-tailed field mouse (Apodemus sylvaticus) (RodentiaMuridae) of Corsica. Bonner Zoologische Beiträge 44: 147-163.

Lomolino MV. 2005. Body size evolution in insular vertebrates: generality of the island rule. Journal of Biogeography 32: 1683-1699.
Lomolino MV, Sax DF, Palombo MR, van der Geer AA. 2012. Of mice and mammoths: evaluations of causal explanations for body size evolution in insular mammals. Journal of Biogeography 39: 842-854.

Magnanou E, Fons R, Blondel J, Morand S. 2005. Energy expenditure in Crocidurinae shrews (Insectivora): Is metabolism a key component of the insular syndrome? Comparative Biochemistry and Physiology Part A 142: 276285.

McDevitt AD, Montgomery WI, Tosh DG, Lusby J, Reid N, White TA, McDevitt D, O'Halloran J, Searle JB, Yearsley JM. 2014. Invading and expanding: range dynamics and ecological consequences of the greater white-toothed shrew (Crocidura russula) invasion in Ireland. PLOS One 9: e100403.

Michaux JR, de Bellocq JG, Sarà M, Morand S. 2002. Body size increase in insular rodent populations: a role of predators? Global Ecology \& Biogeography 11: 427-436.

Millien V. 2011. Mammals evolve faster on smaller islands. Evolution 65: 1935-1944.

Moncunill-Solé B, Jordana X, Köhler M. 2016. How common is gigantism in insular fossil shrews? Examining the 'Island Rule' in soricids (Mammalia: Soricomorpha) from Mediterranean Islands using new body mass estimation models. Biological Journal of the Linnean Society 178: 163-182.

Monteiro LR. 1999. Multivariate regression models and geometric morphometrics: the search for causal factors in the analysis of shape. Systematic Biology 48: 192-199.

Montgomery WI, Montgomery SSJ, Reid N. 2015. Invasive alien species disrupt spatial and temporal ecology and threaten extinction in an insular, small mammal community. Biological Invasions 17: 179-189.

Palmer AR, Strobeck C. 1986. Fluctuating asymmetry: measurement, analysis, patterns. Annual Reviews in Ecology and Systematics 17: 391-421.

Raia P, Meiri S. 2011. The tempo and mode of evolution: body sizes of island mammals. Evolution 65: 1927-1934.

Rohlf FJ. 2010. tpsDig. Version 2.16. Department of Ecology and Evolution, State University of New York at Stony Brook.

Ros J, Olivella I, Gili JM. 1984. Els sistemes naturals de les Illes Medes. Barcelona: Institut d'Estudis Catalans.

Russell RC, Ringler D, Trombini A, LeCoree M. 2011. The island syndrome and population dynamics of introduced rats. Oecologia 167: 667-676.

Sánchez-Chardi A, Nadal J. 2007. Bioaccumulation of metals and effects of a landfill in small mammals. Part I. The greater white-toothed shrew, Crocidura russula. Chemosphere 68: 703-711.

Sánchez-Chardi A, López-Fuster MJ. 2009. Metal and metalloid accumulation in shrews (Soricomorpha, Mammalia) from two protected Mediterranean coastal sites. Environmental Pollution 157: 1243-1248.

Sánchez-Chardi A, López-Fuster MJ, Nadal J. 2007. Bioaccumulation of lead, mercury, and cadmium in the greater white-toothed shrew, Crocidura russula, from the Ebro Delta (NE Spain): sex- and age-dependent variation. Environmental Pollution 145: 7-14.

Sánchez-Chardi A, Peñarroja-Matutano C, Borrás M, Nadal J. 2009. Bioaccumulation of metals and effects of a 
landfill in small mammals. Part III. Structural alterations. Environmental Research 109: 960-967.

Sánchez-Chardi A, García Pando M, López-Fuster MJ. 2013. Chronic exposure to environmental stressors induces fluctuating asymmetry in shrews inhabiting protected Mediterranean sites. Chemosphere 93: 916-923.

Tamarin RH. 1978. Dispersal, population regulation and K-selection in field mice. The American Naturalist 112: 545555.

VanValen LM. 1973. A new evolutionary law. Evolutionary Theory 1: 1-30.

Ventura J, López-Fuster MJ. 2000. Morphometric analysis of the black rat, Rattus rattus, from Congreso Island (Chafarinas Archipelago, Spain). Orsis 15: 91-102.

White TA, Searle J. 2007a. Factors explaining increased body size in common shrews (Sorex araneus) on Scottish islands. Journal of Biogeography 34: 356-363.

White TA, Searle J. 2007b. Genetic diversity and population size: island populations of the common shrew, Sorex araneus. Molecular Ecology 16: 2005-2016.

White TA, Searle J. 2008. Mandible asymmetry and genetic diversity in island populations of the common shrew, Sorex araneus. Journal of Evolutionary Biology 21: 636-641.

White TA, Searle J. 2009. Ecomorphometric variation and sexual dimorphism in the common shrew (Sorex araneus). Journal of Evolutionary Biology 22: 1163-1171.

Young RL, Haselkorn TS, Badyaev AV. 2007. Functional equivalence of morphologies enables morphological and ecological diversity. Evolution 61: 2480-2492.

Young RL, Sweeney MJ, Badyaev AV. 2010. Morphological diversity and ecological similarity: versatility of muscular and skeletal morphologies enables ecological convergence in shrews. Functional Ecology 24: 556-565.

Received: 28 February 2018

Revised and accepted: 15 November 2018

Published online: 30 November 2018

Editor: A. Ivanović

\section{On-line Supplementary Information (SI)}

Figure S1. Body mass (g) of C. russula specimens for each combination of site and sex. Small empty circles indicate individual data, the large circles indicate the mean mass for each combination, and lines indicate their $95 \%$ confidence intervals (CIs) for the mean. CIs were calculated after 1000 alternative means of randomly permutated data with replacement. 